\title{
Modelling for Vehicle to Vehicle Applications
}

\author{
David I. Laurenson $^{\# 1}$, Michail Matthaiou ${ }^{\dagger 2}$, Xiang Cheng ${ }^{* 3}$, Cheng-Xiang Wang ${ }^{* 4}$ \\ \# Joint Research Institute for Signal \& Image Processing, Institute for Digital Communications \\ The University of Edinburgh, Kings Buildings, Edinburgh, EH9 3JL \\ ${ }^{1}$ Dave.Laurenson@ed.ac.uk \\ ${ }^{\dagger}$ Institute for Circuit Theory and Signal Processing, Technische Universität München (TUM) \\ Arcistrasse 21, 80333, Munich, Germany \\ 2matthaiou@nws.ei.tum.de \\ ${ }^{*}$ Joint Research Institute for Signal \& Image Processing, School of Engineering \& Physical Sciences \\ Heriot-Watt University, Edinburgh, EH14 $4 A S$ \\ ${ }^{3}$ xc48@hw.ac.uk, ${ }^{4}$ cheng-xiang.wang@hw.ac.uk
}

\begin{abstract}
Rapid growth of personal communications has led to the development of many wireless systems, competing for radio bandwidth. In the vehicular environment, there is recognition of the importance of communications, and therefore the ability to model these channels is important. This paper addresses this issue through the exploration of both vehicle to roadside (VTR) and vehicle to vehicle (VTV) communications. The paper presents a design methodology for achieving the maximum capacity under the above mentioned propagation conditions as well as reference and stochastic simulation models for vehicular environments.
\end{abstract}

\section{INTRODUCTION}

Mobile communication, in all its forms, has become a vital element of today, and the concept of "always/everywhere connected" is fast becoming a reality. With this concept is the expectation that devices other than mobile phones require connectivity to a wider Internet, whether this be autonomous monitoring equipment, public transport vehicles, or even private vehicles. To this end, the issue of vehicle to vehicle (VTV) communication is becoming relevant, whether it be for safety purposes [1] or for entertainment [2]; hence, it is necessary to explore the vehicle to roadside (VTR) and VTV links in detail.

To increase capacity in home and office environments, multiple-input multiple-output (MIMO) technology has become popular, however this technology does not perform well in many line-of-sight (LoS) scenarios, due to the lack of scattering. However, recent research in the area of short-range communications has demonstrated that high capacities are still achievable, even where a LoS exists [3],[4], as long as specifically designed antenna arrays are deployed at both the transmitter (Tx) and receiver (Rx). Unfortunately, these papers are tied to fixed-to-mobile communication systems, and thus the results are not immediately transferable to the vehicular environment. This paper expands on work presented in [5], which explored the effect of non-optimal separation of communicating terminals in a VTR scenario; in the following, a VTV scenario is also investigated where the effects of antenna spacings and terminal heights are taken into account. This acknowledges the practical issue that it is not possible to optimise antenna spacing for VTV communication due to the mobile nature of the terminals; however it is important to maximise capacity for VTR links.

In addition to the deterministic LoS component, and its ground reflection, that are dominant in VTV communication, there is a requirement to also model the scattering environment that such links experience. Existing fixed-tomobile scattering reference models generally assume that the $\mathrm{Tx}$ and $\mathrm{Rx}$ experience different scattering due to their different locations and mobility patterns. However, in a VTV scenario, the same scattering environment will be experienced by both, and both entities are in motion, and such reference models are not applicable. Therefore, a two-ring reference model was proposed in [6] and has been widely used to mimic $\mathrm{V} 2 \mathrm{~V}$ channels due to its good trade-off between the model accuracy and mathematical tractability.

Since reference models assume an infinite number of harmonic functions, they cannot be implemented directly in practice. Accurate simulation models play a major role in the practical simulation and performance evaluation of $\mathrm{V} 2 \mathrm{~V}$ systems. When generating stochastic simulation models, a sum-of-sinusoids (SoS) approach is commonly used to approximate the underlying fading process through the superposition of a finite number of harmonic functions, in contrast to reference models. Based on the two-ring reference model, a new stochastic SoS simulator was proposed in [6] for isotropic V2V Rayleigh fading channels. This stochastic simulator was further improved in [7] by the introduction of orthogonal functions for in-phase and quadrature components, and in [8] by the inclusion of a line-of-sight component. To date, the only published work on stochastic SoS simulation models for non-isotropic V2V Rayleigh fading channels is [9], with our paper [10] yet to appear in print. Section III of this paper extracts relevant sections of [10] to complete the model for VTV communication.

\section{CAPACITY ANALYSIS}

In vehicle to vehicle, or vehicle to roadside communications, the channel is typically dominated by a LoS component giving rise to Ricean fading statistics instead of Rayleigh. Thus, when optimising antenna positioning and antenna element spacing for MIMO communications, the 


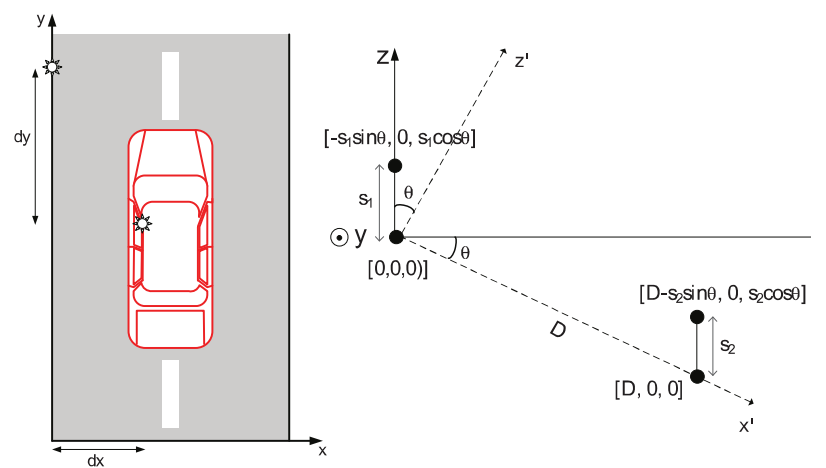

Fig. 1 VTR antenna configuration.

deterministic component must be considered. The effect of the non-deterministic component, combined with the deterministic one, should be considered after the system has been optimised.

\section{A. Vehicle to Roadside (VTR) system optimisation}

Consider the scenario in Fig. 1 where a vehicle is in communication with a roadside mounted station. The distance, $D$, between the vehicle and the roadside station varies as the vehicle moves, whilst the height difference between the two, and this is expressed in terms of a rotation angle $\theta$, which also varies with displacement.

For the purposes of calculation, a transformation to the axes $z^{\prime}$ and $x$ ' can be made, which simplifies some of the calculation. The inter-element distances, which are vertically separated, at the roadside and on the vehicle terminal are $s_{1}$ and $s_{2}$ respectively. The distance between the first (lower) element of each array is $D=\sqrt{d x^{2}+d y^{2}+d z^{2}}$, and the rotation angle given by $\theta=\sin ^{-1}\left(\frac{d z}{D}\right)$.

For the $2 \times 2$ MIMO configuration used, the channel can be expressed as a $2 \times 2$ matrix, $\mathbf{H}$, with elements corresponding to the transfer function between corresponding pairs of antennae. Assuming flat fading, the matrix can be re-expressed as a deterministic component, $\mathbf{H}_{\mathrm{L}}$, and a non-deterministic one, $\mathbf{H}_{\mathrm{W}}$, as [5]

$$
\mathbf{H}=\sqrt{\frac{K}{K+1}} \mathbf{H}_{\mathrm{L}}+\sqrt{\frac{1}{K+1}} \mathbf{H}_{\mathrm{W}}
$$

where $K$ is the Ricean $\mathrm{K}$-factor, denoting the ratio of the power of the deterministic component to the total power of the scattered paths. Without loss of generality, we can assume ideal isotropic radiators, and negligible differences in pathlosses; then, the normalised free-space matrix for the deterministic component becomes

$$
\mathbf{H}_{\mathrm{L}}=\left[\begin{array}{ll}
e^{-j k d_{1,1}} & e^{-j k d_{1,2}} \\
e^{-j k d_{2,1}} & e^{-j k d_{2,2}}
\end{array}\right]
$$

where $k=2 \pi / \lambda$ is the wavenumber corresponding to the carrier wavelength, $\lambda$, and $d_{m, n}$ is the distance between antenna elements $m$ of the vehicle and $n$ of the roadside.
Using the Taylor series approximation

$$
\sqrt{(D+\mu)^{2}+v^{2}} \approx(D+\mu)+\frac{v^{2}}{2(D+\mu)},
$$

which is valid provided $D+\mu \gg>$, the distances between the elements can be expressed as

$$
\begin{aligned}
& d_{1,1}=D \\
& d_{1,2} \approx D+s_{1} \sin \theta+\frac{\left(s_{1} \cos \theta\right)^{2}}{2\left(D+s_{1} \sin \theta\right)} \\
& d_{2,1} \approx D-s_{2} \sin \theta+\frac{\left(s_{2} \cos \theta\right)^{2}}{2\left(D-s_{2} \sin \theta\right)} \\
& d_{2,2} \approx D+\left(s_{1}-s_{2}\right) \sin \theta+\frac{\left(\left(s_{1}-s_{2}\right) \cos \theta\right)^{2}}{2\left(D+\left(s_{1}-s_{2}\right) \sin \theta\right)}
\end{aligned}
$$

By making a further simplification, after noting that $s_{i} \sin \theta$ is significantly smaller than $D$, the denominators of the fractions can be written as $2 D$.

The capacity of the deterministic system can be written as

$$
C=\log _{2}\left(\operatorname{det}\left(\mathbf{I}_{2}+\frac{\rho}{2} \mathbf{H}_{\mathrm{L}} \mathbf{H}_{\mathrm{L}}^{\mathrm{H}}\right)\right)
$$

where $(.)^{\mathrm{H}}$ denotes the Hermitian transpose operation. Using the maximisation criteria in [11], and substituting in the simplified form of (3), the maximum of $C$ can be found when

$$
s_{1} s_{2} \approx \lambda\left(r+\frac{1}{2}\right) \frac{D}{\cos ^{2} \theta}, \quad r \in \mathbf{Z}^{+}
$$

where $\mathbf{Z}^{+}$denotes the set of positive integers.

Thus, the optimal antenna spacing is dependent upon the distance between the transmitter and the receiver, as well as the rotation angle. The further apart the vehicle and roadside station are, and the larger the height difference, the further apart the antenna elements need to be to maximise capacity.

\section{B. Sub-optimal antenna spacing}

In a practical system, antenna spacing is fixed, thus the separation is, by necessity, not optimal for the majority of separations. To illustrate this effect, the VTR system is optimised for the position when $d x=5 \mathrm{~m}, d y=0$, and $d z=$ $2 \mathrm{~m}$, i.e. when the vehicle is directly beside the roadside station. In this configuration, using the solution of equal interelement spacing, the minimum separation is found to be when $s_{1}=s_{2}=39.85 \mathrm{~cm}$. For a system SNR of $20 \mathrm{~dB}$, a maximum capacity of $13.32 \mathrm{bits} / \mathrm{s} / \mathrm{Hz}$ is achieved by this system. The capacity of this system will now be investigated using this antenna configuration.

\section{1) Vehicle to roadside}

Fig. 2 shows the capacity variation of the system as the separation distance between the vehicle and the roadside changes. It is clear that the capacity is sensitive to displacement from the roadside, with the maximum capacity being achieved at small displacements from the roadside. Examining the situation for larger displacements, capacities in excess of $8 \mathrm{bits} / \mathrm{s} / \mathrm{Hz}$ can still be achieved at a separation of 
$50 \mathrm{~m}$. In the region where the separation distance is less than $20 \mathrm{~m}$, the achievable capacity is in excess of the Rayleigh

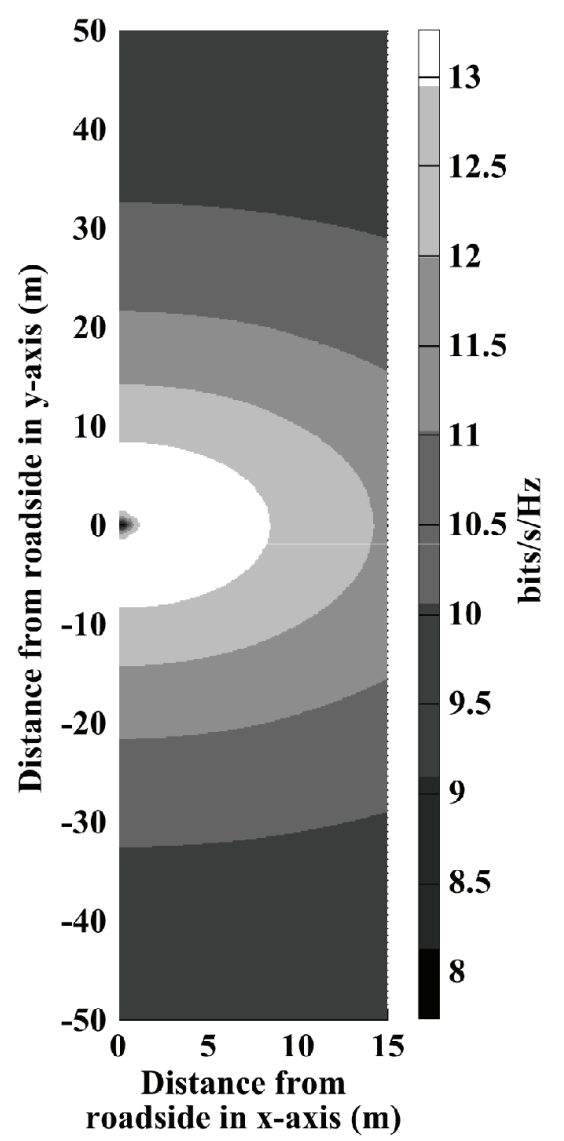

Fig. 2: Capacity variation against displacement on the $x y$ plane.

capacity of $11.4 \mathrm{bits} / \mathrm{s} / \mathrm{Hz}$ for the same SNR. This observation is consistent with results in [12] where different optimised architectures are proposed for near-field LoS propagation. component of the propagation.

\section{2) Vehicle to vehicle}

It is anticipated that VTV communications will become an increasingly important component in the future as safety systems, and infotainment systems, are proposed for the vehicular environment. Thus, the antenna configuration that is optimised for VTR communications should also support VTV communications. The effect of vehicle displacement, as well as mounting height of the antenna, will be significant factors in determining the capacity of the resulting communication links.

Fig. 3 shows the parameters used in the VTV scenario. The antenna separation is assumed to be identical, and fixed at the separation that is optimal for VTR communication, as is the height from the ground. On a highway environment, the effect of the ground cannot be ignored as it forms a highly reflective surface at grazing angles. For the sake of simplicity, a ground reflection coefficient, $\alpha$, is assumed to be -1 for all vehicle separations as $D>h$. Each deterministic channel

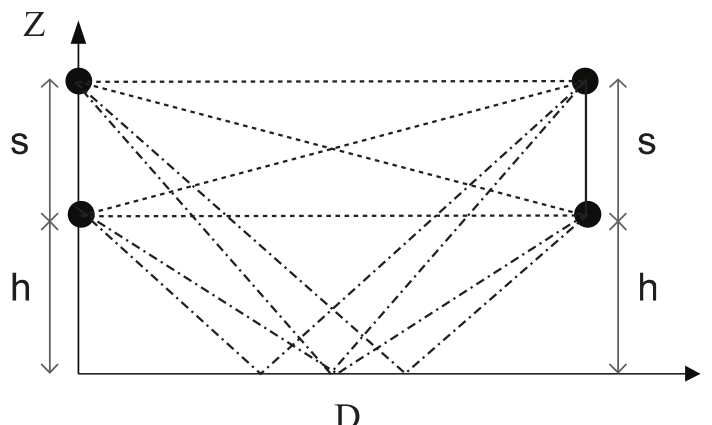

Fig. 3: VTV communication configuration.

therefore consists of two waves - a direct wave and a reflected one.

To determine the capacity of this system, the channel matrix becomes:

$$
\mathbf{H}_{\mathrm{L}}=\frac{1}{\sqrt{2}}\left[\begin{array}{ll}
e^{-j k d_{1,1}}+e^{-j k d_{1,1}^{\prime}} & e^{-j k d_{1,2}}+e^{-j k d_{1,2}^{\prime}} \\
e^{-j k d_{2,1}}+e^{-j k d_{2,1}^{\prime}} & e^{-j k d_{2,2}}+e^{-j k d_{2,2}^{\prime}}
\end{array}\right]
$$

with

$$
\begin{aligned}
& d_{1,1}=D ; \quad d_{1,1}^{\prime}=\sqrt{4(s+h)^{2}+D^{2}} \\
& d_{1,2}=\sqrt{s^{2}+D^{2}} ; \quad d_{1,2}^{\prime}=\sqrt{(s+2 h)^{2}+D^{2}} \\
& d_{2,1}=\sqrt{s^{2}+D^{2}} ; \quad d_{2,1}^{\prime}=\sqrt{(s+2 h)^{2}+D^{2}} \\
& d_{2,2}=D ; \quad d_{2,2}^{\prime}=\sqrt{4 h^{2}+D^{2}} .
\end{aligned}
$$

Substituting (6) and (7) into (4), results in the capacity of the system. Unfortunately, although the resulting equation is exact, it cannot be expressed in a compact form, and thus is not presented in this paper. However, it is possible to evaluate the equation using an algebraic solver, such as Maple, and produce meaningful results from this evaluation. In order to determine the system capacity for the optimum spacings obtained above, this was performed, and the resulting capacity graph is presented in Fig. 4 below.

From this figure two trends can be observed. As the distance between the two vehicles increases from $50 \mathrm{~m}$ to $600 \mathrm{~m}$, the capacity falls, with a constant SNR, $\rho$, of $20 \mathrm{~dB}$ being maintained. The capacity is also critically dependent upon the height of the antennas. When mounted on the base of the vehicle, the capacity is low, whereas mounting them at the roof height of the vehicle gives rise to the highest capacity figures. This would imply that rooftop mounting of antennas for VTV scenarios is likely to give rise to the highest capacities.

Close examination of Fig. 4 will also reveal that at the lowest vehicle separations plotted, when the antenna is mounted at roof height, some fluctuations in capacity can be observed. Fig. 5 is a plot of the capacity for a fixed antenna height of $1.4 \mathrm{~m}$ for various vehicle separations. It can be clearly observed that at low separations the fluctuations become pronounced. The significant decrease in capacity at $25 \mathrm{~m}$ results from phase cancellation by the reflected 


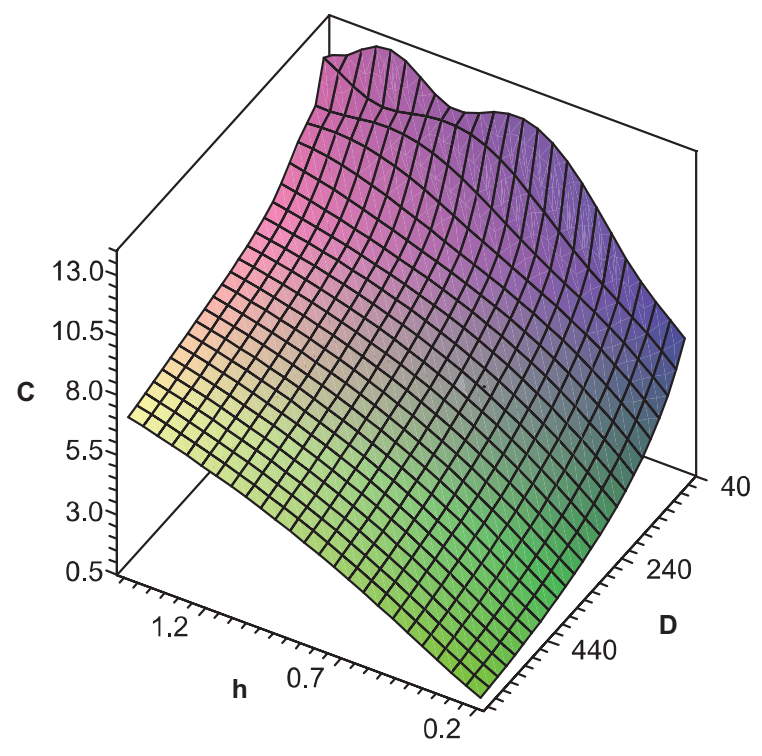

Fig. 4: Capacity of a VTV channel for different antenna heights and vehicle separations.

component from the road surface. However, as the reflection coefficient, $\alpha$, deviates from -1 at small vehicle separations due to the higher angle of incidence, the practical system will not observe the same depth of null at this distance. Results are not presented for smaller separations as not only does $\alpha$ deviate from -1 , but in a mobile environment, vehicles are unlikely to be communicating over such short distances.

\section{Effect of non-deterministic components}

In order to determine the effect of scattering, $\mathbf{H}_{\mathrm{W}}$ must be considered. In this paper, $\mathbf{H}_{\mathrm{W}}$ is assumed to consist of complex Gaussian elements of unity variance that are independent from each other. The $K$-factor of (1) is used as a measure of the balance between deterministic and nondeterministic components. To demonstrate the effect of the non-deterministic component, the two scenarios of the VTR and VTV scenario are evaluated for a total of 50,000 MonteCarlo realisations of $\mathbf{H}$, and the capacity of each of these is combined to form a CDF of the system capacity. In the VTV scenario the separation is selected to be $200 \mathrm{~m}$ as a typical range of communication, and an antenna height of $1.4 \mathrm{~m}$ chosen to maximise capacity. Fig. 6 shows the capacity of the optimal VTR scenario as a function of the $K$ factor. As the $K$ factor increases, MIMO capacity yields a monotonic increase while diversity level is also enhanced (steeper CDF curves). Fig. 7 presents the results for the VTV scenario using the antenna spacing that is optimised for VTR communication

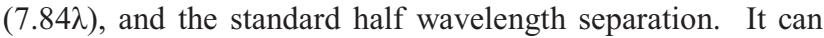
be seen that with an increase in $K$, although capacity initially increases for both configurations, it then falls lower than the Rayleigh i.i.d. channel to converge on the capacity indicated in Fig. 5. It is satisfying to note, though, that the capacity of the VTR optimised configuration is higher than that of the

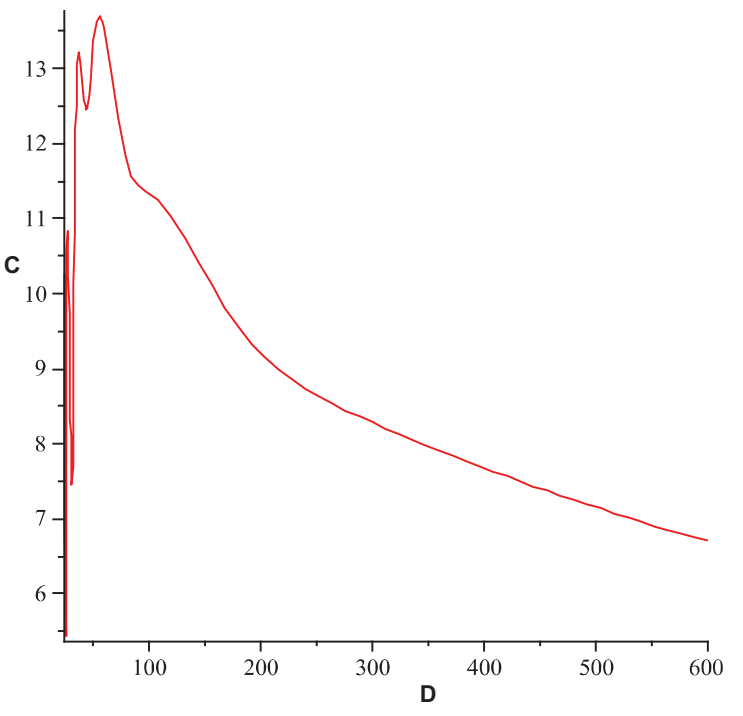

Fig. 5: Capacity of a VTV channel with an antenna height of $1.4 \mathrm{~m}$.

standard configuration. As the $K$ factor decreases to $-\infty \mathrm{dB}$, i.e. the deterministic component disappears, the capacity degrades to that of the Rayleigh i.i.d. channel.

As indicated, the results for the VTR situation, indicating that higher $K$ factors result in capacity increases, are in line with results presented in $[3,4,11,12]$. However, as the mobile separation increases between communicating vehicles, the capacity falls below that of the Rayleigh case as the channels become more correlated. Thus, the system fails to realise these promised gains when the antenna positioning cannot be optimised for the VTV scenario.

In order to determine the performance of a practical system, it is necessary to consider the communication requirements. It is recognised that VTR communication requires a high capacity as mobiles may be involved in relaying data for other mobile users. Hence the VTR link may have the highest data requirements. Thus, although the antenna configuration optimised for the VTR link is not optimal for a VTV link, the spacing may nevertheless be the appropriate one for a full system deployment. The analysis, and results, presented here can be used in the assessment of a complete system to ensure that sufficient capacity is available for the appropriate links.

\section{MODELLING OF SCATTERING}

In the previous section, an analysis of the deterministic component between a vehicle and a roadside, as well as a VTV, is presented, and used to optimise antenna configuration for vertically mounted MIMO systems. This section will briefly describe a reference model and focus on the elaboration of the corresponding stochastic simulation model for VTV communication that accounts for multiple scattering in the environment. 


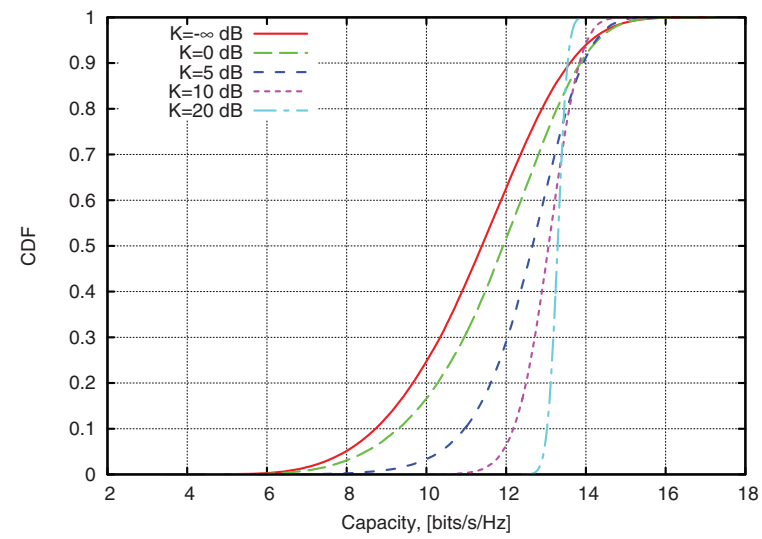

Fig. 6: Capacity curves for MIMO vehicle to roadside channel

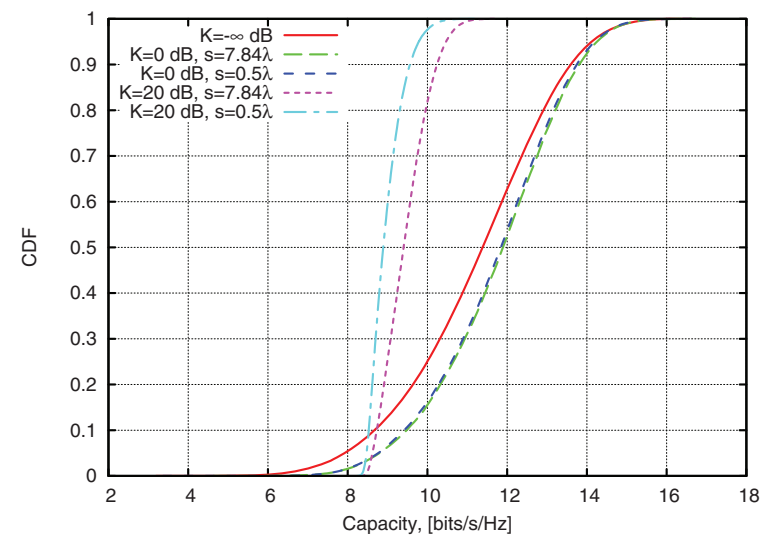

Fig. 7: Capacity curves for MIMO channels at a vehicle separation of

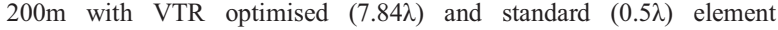
separations.

\section{A. Reference model}

The scattering environment for VTV communications is quite distinct from that of traditional cellular links where the base station typically is not surrounded by scatterers in macrocell environments. In the vehicular environment, both the Tx and $\mathrm{Rx}$ experience similar local scattering statistics, which can be modelled as two rings of effective scatterers, one around each mobile [6]. The scenario is depicted in Fig. 8 with two mobiles travelling with velocities $v_{\mathrm{T}}$ and $v_{\mathrm{R}}$ in directions $\gamma_{\mathrm{T}}$ and $\gamma_{R}$ respectively. The scatterers are assumed to lie on circles centred on the mobiles.

Based upon the aforementioned VTV two-ring reference model as shown in Fig. 8, we can express the complex fading envelope under a narrowband non-isotropic scattering Rayleigh fading assumption as:

$h(t)=\lim _{N, M \rightarrow \infty} \frac{1}{\sqrt{N M}} \sum_{n, m=1}^{N, M} e^{j \psi_{n m}} e^{j\left[2 \pi f_{T_{\max }} t \cos \left(\phi_{T}^{m}-\gamma_{T}\right)+2 \pi f_{R_{\max }} t \cos \left(\phi_{R}^{n}-\gamma_{R}\right)\right]}$

where $N$ and $M$ denote the number of harmonic functions representing scatterers located on the ring around the receiver

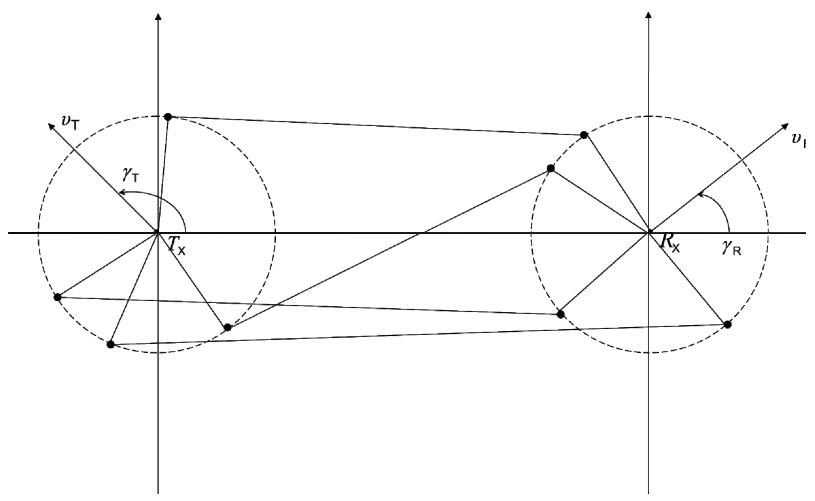

Fig. 8: Layout for a VTV two-ring reference model

and transmitter, respectively, $f_{T_{\max }}$ and $f_{R_{\max }}$ are the maximum Doppler frequencies of the mobiles, $\phi_{T}^{m}$ and $\phi_{R}^{n}$ are the angle of departure and angle of arrival of the $m^{\text {th }}$ and $n^{\text {th }}$ path, respectively, and $\psi_{n m}$ is a random phase.

Under the limit, the angles of departure and arrival can be expressed using a continuous distribution. For the reference model the von Mises distribution [13] is used to characterise angles of departure and arrival as it can model both commonly used distributions as well as measured data very well. Upon applying this distribution [13], the correlation function for this reference model can be expressed as:

$$
\begin{aligned}
& \rho_{h h}(\tau)=\frac{1}{I_{0}\left(k_{T}\right) I_{0}\left(k_{R}\right)} I_{0}\left[\sqrt{A_{T}^{2}+B_{T}^{2}}\right] I_{0}\left[\sqrt{A_{R}^{2}+B_{R}^{2}}\right] \\
& A_{T}=k_{T} \cos \left(\mu_{T}\right)+j 2 \pi \tau f_{T_{\max }} \cos \left(\gamma_{T}\right) \\
& B_{T}=k_{T} \sin \left(\mu_{T}\right)+j 2 \pi \tau f_{T_{\max }} \sin \left(\gamma_{T}\right) \\
& A_{R}=k_{R} \cos \left(\mu_{R}\right)+j 2 \pi \tau f_{R_{\max }} \cos \left(\gamma_{R}\right) \\
& B_{R}=k_{R} \sin \left(\mu_{R}\right)+j 2 \pi \tau f_{R_{\max }} \sin \left(\gamma_{R}\right)
\end{aligned}
$$

where $I_{0}$ is the modified Bessel function of the $0^{\text {th }}$ order, and $\mu_{T}$ and $\mu_{R}$ are the mean angles of $\phi_{T}$ and $\phi_{R}$ respectively.

\section{B. Stochastic simulation model}

The reference model presented above describes the statistical properties of the system that is being considered, however due to its infinite complexity $(\{N, M\} \rightarrow \infty)$ the reference model cannot be implemented directly in practice. Therefore, stochastic simulation models are required to allow performance evaluations to be carried out. In contrast to the reference model in (8), the stochastic SoS simulation model is expressed as a summation of a finite number of scatterers as follows: 


$$
\begin{aligned}
& \hat{h}(t)=\hat{h}_{i}(t)+j \hat{h}_{q}(t) \\
& \hat{h}_{i}(t)=\frac{1}{\sqrt{N_{i} M_{i}}} \sum_{n_{i}, m_{i}=1}^{N_{i}, M_{i}} \cos \left[\hat{\psi}_{n_{i}, m_{i}}+2 \pi f_{T_{\max }} t \cos \left(\hat{\phi}_{T}^{m_{i}}-\gamma_{T}\right)+2 \pi f_{R_{\max }} t \cos \left(\hat{\phi}_{R}^{n_{i}}-\gamma_{R}\right)\right] \\
& \hat{h}_{q}(t)=\frac{1}{\sqrt{N_{q} M_{q}}} \sum_{n_{q}, m_{q}=1}^{N_{q}, M_{q}} \cos \left[\hat{\psi}_{n_{q}, m_{q}}+2 \pi f_{T_{\max }} t \cos \left(\hat{\phi}_{T}^{m_{q}}-\gamma_{T}\right)+2 \pi f_{R_{\max }} t \cos \left(\hat{\phi}_{R}^{n_{q}}-\gamma_{R}\right)\right]
\end{aligned}
$$

where the hat denotes a random variable corresponding to the unhatted variable in the reference model.

The principal issue for design of the stochastic simulation model in (10) is to design the angles, $\hat{\phi}_{T}^{m_{i / q}}$ and $\hat{\phi}_{R}^{n_{i / q}}$, to guarantee that the simulation model can reproduce the desired statistical properties of the reference model as faithfully as possible with reasonable complexity, i.e., with a finite number of $N_{i / q}$ and $M_{i / q}$. To this end, the method of Zheng and Xiao [14] for isotropic scattering cellular channels is used. However, unlike isotropic scattering environments, the appropriate range on which the angles of departure and arrival are designed is different for non-isotropic VTV channels. In order to generate an efficient simulation model, the uniqueness of the sine and cosine functions in (10) should be fulfilled. This means the following conditions should be met:

$$
\begin{array}{ll}
\cos \left(\hat{\phi}_{T}^{m_{i / q}}-\gamma_{T}\right) \neq \cos \left(\hat{\phi}_{T}^{m_{i / q}^{\prime}}-\gamma_{T}\right) ; & m_{i / q} \neq m_{i / q}^{\prime} \\
\cos \left(\hat{\phi}_{R}^{n_{i / q}}-\gamma_{R}\right) \neq \cos \left(\hat{\phi}_{R}^{n_{i / q}^{\prime}}-\gamma_{R}\right) ; & n_{i / q} \neq n_{i / q}^{\prime} .
\end{array}
$$

It is difficult to obtain sets of angles that meet these two conditions over all non-isotropic environments, therefore the scenario is divided up into three cases in order to perform the design.

\section{1) Case 1: Alignment of components}

In this case, the directions of travel are $0^{\circ}$, as are the mean angles of departure and arrival, thus $\mu_{T}=\gamma_{T}=\mu_{R}=\gamma_{R}=0$ or $\pi$. Here it is possible to set $N_{i}=N_{q}=N$, and $M_{i}=M_{q}=M$. Assuming that the inverse of the cumulative distribution functions of the angle of arrival and angle of departure exist, and are denoted by $F_{R}^{-1}$ and $F_{T}^{-1}$ respectively, then the random deviates can be defined as:

$$
\begin{aligned}
& \hat{\phi}_{R}^{n_{i / q}}=F_{R}^{-1}\left(\frac{n_{i / q}-1 / 2+\theta_{R}}{N}\right), \quad n_{i / q}=1 . . N \\
& \hat{\phi}_{T}^{m_{i / q}}=F_{T}^{-1}\left(\frac{m_{i / q}-1 / 2+\theta_{T}}{M}\right), \quad m_{i / q}=1 . . M
\end{aligned}
$$

where $\theta_{R}$ and $\theta_{T}$ are independent uniform random deviates distributed in the interval $[-0.5,0.5)$. For this case it is sufficient that $\hat{\phi}_{T}^{m_{i / q}}$ and $\hat{\phi}_{R}^{n_{i / q}}$ lie in the range $[0, \pi)$.

\section{2) Case II: The general non-aligned case}

In this situation, which excludes cases I and III (which is dealt with below), it is necessary to extend the range of values for $\hat{\phi}_{T}^{m_{i / q}}$ and $\hat{\phi}_{R}^{n_{i / q}}$ such that they lie in the range $[-\pi, \pi)$. Apart from this distinction, an identical formulation to Case I can be made.

3) Case III: The mean propagation angle is perpendicular to the direction of travel

For this case, $\left|\mu_{T}-\gamma_{T}\right|=\left|\mu_{R}-\gamma_{R}\right|=\frac{\pi}{2}$, which requires special consideration as the cross-correlation between the inphase and quadrature components of $h(t)$ should be zero. In order to guarantee this situation, the simple step of setting $M_{i} \neq M_{q}$ and $N_{i} \neq N_{q}$ is sufficient. This step simplifies the process of angle generation which would otherwise have to take account of this requirement in the design. Similar to Case II, the full range of $[-\pi, \pi)$ is necessary for the design of the angles of departure and arrival for this case.

The correlation function for the three cases can be derived analytically, with the full derivation being presented in [10]. To illustrate the effectiveness of the model, Fig. 9 shows a comparison between the stochastic simulation model and the reference model for the following parameters: a maximum Doppler frequency of $100 \mathrm{~Hz}, N=M=10$ for Cases $I$ and $I I$, $N_{i}=M_{i}=10, N_{q}=M_{q}=11$ for Case III, $\gamma_{T}=\gamma_{R}=0$ and a normalised sampling period $f_{T_{\max }} T_{s}=0.005 . \quad\left(T_{s}\right.$ is the sampling period). Results presented in the figure are averaged over 10 simulation trials.

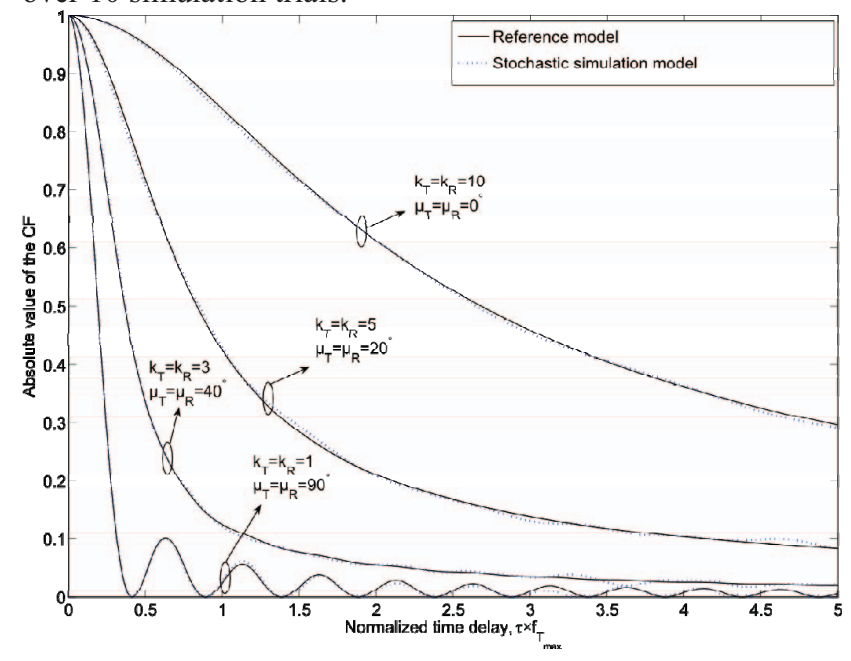

Fig. 9: Comparison between reference and stochastic simulation models.

It is clear from this figure that the stochastic simulation model, with a relatively low number of complex harmonic functions, provides an excellent approximation to the reference model. This implies that it is feasible to model this theoretical reference model in a practical simulation using the stochastic SoS technique, and achieve good agreement with the correlation function of the reference model.

\section{CONCLUSIONS}

This paper has presented two modelling techniques applicable to vehicular environments. The first technique was used to determine the optimum configuration for achieving 
maximum LoS MIMO capacity for VTR MIMO communication systems; the performance of these optimized configurations was then assessed in real-life propagation conditions, such as VTV communications and multipath scattering. Our results have indicated that the optimized VTR configuration could be used for VTV communication as well, however it does not achieve the full MIMO potential of such channels. The analysis has also shown that a high mounting point on a vehicle is advantageous for maximising capacity when a significant ground reflection is present.

The second technique illustrates the means by which an unrealisable reference model for VTV communications can be implemented in practice, retaining the correlation features of the reference model without significant complexity. Numerical results have shown that the introduced stochastic SoS simulation model has an excellent approximation to the correlation properties of the reference model. Therefore, this stochastic simulator can be used for the simulation of future VTV communication systems.

The two techniques, whilst addressing different aspects of the communications link, address fundamental aspects of this communication medium that is increasingly becoming important.

\section{REFERENCES}

[1] M. Röck1, J. Gacnik, and J. Schomerus, "Integration of Car-2-Car communication as a virtual sensor in automotive sensor fusion for advanced driver assistance systems," in Proc. FISITA World Automotive Congress, September 2008.

[2] R. Anthony, A. Leonhardi, C. Ekelin, D. Chen, M. Törngren, G. de Boer, I. Jahnich, S. Burton, O. Redell, A. Weber and V. Vollmer, "A Future Dynamically Reconfigurable Automotive Software System," in Proc. IESS'07, 2007.

[3] F. Bøhagen, P. Orten, and G.E. Øien, "Construction and capacity analysis of high-rank line-of-sight MIMO channels," in Proc. IEEE Wireless Communications and Networking Conference (WCNC), March 2005, pp 432-437.

[4] I. Sarris and A.R. Nix, "Design and performance assessment of highcapacity MIMO architectures in the presence of a line-of-sigh component," IEEE Transactions on Vehicular Technology, vol. 56, no. 4, pp 2194-2202, July 2007.
[5] M. Matthaiou, D.I. Laurenson, and C.-X. Wang, "Capacity study of vehicle-to-roadside MIMO channels with a line-of-sight component," in Proc. Wireless Communications and Networking Conference (WCNC), Las Vegas, USA, March 2008, pp 775-779.

[6] C.S. Patel, G.L. Stüber, and T.G. Pratt, "Simulation of Rayleigh-faded mobile-to-mobile communication channels," IEEE Trans. Commun., vol. 53, no. 11, pp 1876-1884, Nov. 2005

[7] A.G. Zajić and G.L. Stüber, "A new simulation model for mobile-tomobile Rayleigh fading channels," IEEE Wireless Communications and Networking Conference (WCNC), Las Vegas, USA, April 2006, pp 1266-1270.

[8] L.C. Wang, W.C. Liu, and Y.H. Cheng, "Statistical analysis of a mobile-to-mobile Rician fading channel model," IEEE Trans. Veh. Technol., vol. 58, no. 1, pp 32-38, Jan. 2009.

[9] Y.R. Zheng, "A non-isotropic model for mobile-to-mobile fading channel simulations," IEEE MCC'06, Washington, USA, Oct. 2006, pp $1-6$.

[10] X. Cheng, C.-X. Wang, D.I. Laurenson, S. Salous, and A.V. Vasilakos, "New Deterministic and Stochastic Simulation Models for NonIsotropic Scattering Mobile-to-Mobile Rayleigh Fading Channels", to appear in Wireless Communications and Mobile Computing, John Wiley \& Sons, 2009

[11] I. Sarris and A.R. Nix, "Design and performance assessment of maximum capacity MIMO architectures in line-of-sight," IEE Proceedings in Communicaitons, vol. 153, no. 4, pp 482-488, Aug. 2006.

[12] F. Bøhaven, P. Orten and G.E. Øien, "Design of capacity-optimal highrank line-of-sight MIMO channels," IEEE Transactions on Wireless Communications, vol. 6, no. 4, pp 1420-1425, April 2007.

[13] A. Abdi, J.A. Barger, and M. Kaveh, "A parametric model for the distribution of the angle of arrival and the associated correlation function and power spectrum at the mobile station," IEEE Trans. Veh. Techol., vol. 51, no. 3, pp 425-434, May 2002.

[14] Y.R. Zheng and C.S. Xiao, "Improved models for the generation of multiple uncorrelated Rayleigh fading waveforms," IEEE Commun. Lett., vol. 6, no. 6, pp 256-258, Jun. 2002. 